Article

\title{
Vibrational Dynamics in crystalline 4-(dimethylamino) benzaldehyde: Inelastic Neutron Scattering and Periodic DFT Study
}

\author{
Mariela M. Nolasco ${ }^{1, *(D)}$, Paulo J. A. Ribeiro-Claro ${ }^{1}$ (D) and Pedro D. Vaz ${ }^{2}$ (D) \\ 1 CICECO, Departamento de Química, Universidade de Aveiro, P-3810-193 Aveiro, Portugal; prc@ua.pt \\ 2 Champalimaud Foundation, Champalimaud Centre for the Unknown, 1400-038 Lisboa, Portugal; \\ pedro.vaz@fundacaochampalimaud.pt \\ * Correspondence: mnolasco@ua.pt; Tel.: +351-234-370-360
}

Citation: Nolasco, M.M.; Ribeiro-Claro, P.J.A.; Vaz, P.D. Vibrational Dynamics in crystalline 4-(dimethylamino) benzaldehyde: Inelastic Neutron Scattering and Periodic DFT Study. Materials 2022, 15, 475. https://doi.org/10.3390/ ma15020475

Academic Editor: Philippe Colomban

Received: 19 November 2021

Accepted: 5 January 2022

Published: 8 January 2022

Publisher's Note: MDPI stays neutral with regard to jurisdictional claims in published maps and institutional affiliations.

Copyright: (c) 2022 by the authors. Licensee MDPI, Basel, Switzerland. This article is an open access article distributed under the terms and conditions of the Creative Commons Attribution (CC BY) license (https:// creativecommons.org/licenses/by/ $4.0 /)$.

\begin{abstract}
The structure and dynamics of crystalline 4-(dimethylamino) benzaldehyde, 4DMAB are assessed through INS spectroscopy combined with periodic DFT calculations. The excellent agreement between experimental and calculated spectra is the basis for a reliable assignment of INS bands. The external phonon modes of crystalline $4 \mathrm{DMAB}$ are quite well described by the simulated spectrum, as well as the modes involving low-frequency molecular vibrations. Crystal field splitting is predicted and observed for the modes assigned to the dimethylamino group. Concerning the torsional motion of methyl groups, four individual bands are identified and assigned to specific methyl groups in the asymmetric unit. The torsional frequencies of the four methyl groups in the asymmetric unit fall in a region of ca. $190 \pm 20 \mathrm{~cm}^{-1}$, close to the range of values observed for methyl groups bonding to unsaturated carbon atoms. The hybridization state of the $\mathrm{X}$ atom in $\mathrm{X}-\mathrm{CH}_{3}$ seems to play a key role in determining the methyl torsional frequency.
\end{abstract}

Keywords: density functional theory; inelastic neutron scattering; vibrational assignment; molecular crystal; methyl torsion

\section{Introduction}

Molecular interactions lie in a field that is still far from being fully understood. Assessing such molecular interactions unravels understanding of not only its structure but, more importantly, how a system's dynamics contributes at the molecular level to the observable behavior at the macroscopic level. This can be done using a complete toolbox including crystallography (X-ray and neutron), nuclear magnetic resonance and vibrational spectroscopy (infrared and Raman). The latter offer extremely valuable information about the local structural environments of molecules. By using chemical bonds as probes, vibrational spectroscopy provides information about how a given molecule "communicates" with its surroundings. Such vibrational optical spectroscopy techniques can be complemented with the use of inelastic neutron scattering (INS). While IR and Raman are optical spectroscopy techniques relying on optical modes that are symmetry-allowed, INS does not obey the same selection rules, with the intensity of the bands being proportional the incoherent neutron scattering cross-section and corresponding atomic displacements in a given vibrational mode. In INS, the largest scattering cross-section is found for hydrogen, thus making this technique ideal to study organic or other hydrogenous systems. Furthermore, INS is of paramount importance since it makes it possible to study low-wavenumber (and high-amplitude) vibrational modes with high sensitivity, which provides critical structural information about molecular systems that cannot be accessed using IR or Raman.

The power of INS to study condensed systems is strongly and accurately complemented by the use of periodic density functional theory (periodic DFT) calculations in a cooperative fashion [1-10]. DFT methods, whether discrete or periodic, offer extreme 
and valuable assistance by predicting vibrational normal-mode eigenvectors. However, periodic methods offer unquestionable further advantage over their discrete counterparts by predicting those vibrational modes found in crystalline systems, such as crystal field splitting and molecular librational and translational modes, which are observed experimentally with INS. Therefore, the combination of INS with periodic DFT methods yields a very accurate yet convenient systematic approach to test molecular models and explain molecular interaction phenomena.

In this work, we present a study where the INS spectrum of 4-(dimethylamino) benzaldehyde (4DMAB) is assessed, and its insights are supported by periodic DFT calculations. $4 \mathrm{DMAB}$ closes a sequence of benzaldehyde derivatives, the INS spectra of which were obtained prior to the determination of their crystal structure (within a related project with $\mathrm{C}-\mathrm{H} \cdots \mathrm{O}$ hydrogen bonds) and are now being used to assess the description of vibrational spectra from combining INS spectroscopy with periodic DFT calculations $[4,9,10]$. In the INS spectrum of $4 \mathrm{DMAB}$, the low-wavenumber region $\left(<300 \mathrm{~cm}^{-1}\right)$ is dominated by modes arising from the substituent groups of the phenyl ring, namely benzaldehyde, amine and its methyl group moieties beyond the translational and librational modes. The presence of band splitting and the dynamics of methyl torsions in the crystal were confirmed both experimentally and computationally and are presented and discussed.

\section{Materials and Methods}

4-(dimethylamino) benzaldehyde was obtained commercially at high-purity grade (Sigma-Aldrich, CAS number 100-10-7, 99\% purity, St. Louis, MO, USA) and used without further purification.

Inelastic neutron scattering spectroscopy: The INS spectrum was obtained using an indirect geometry time-of-flight spectrometer, TOSCA, at the ISIS Neutron and Muon Source at the Rutherford Appleton Laboratory (Chilton, UK) [11,12]. The sample of 4(dimethylamino) benzaldehyde, with a total amount of ca. $2 \mathrm{~g}$, was loaded into a flat, thin-walled aluminium can of $4.8 \mathrm{~cm}$ height $\times 4 \mathrm{~cm}$ width $\times 2 \mathrm{~mm}$ path length, sealed with an indium wire and mounted perpendicularly to the beam by using a regular TOSCA center stick. The INS spectrum, measured for the 16 to $8000 \mathrm{~cm}^{-1}$ energy-transfer range, was recorded below $15 \mathrm{~K}$ with a resolution of $\triangle \mathrm{E} / \mathrm{E} \approx 1.5 \%$. MANTID program (version 4.0.0) [13] was used to convert the data to the to the conventional scattering law, $S(Q, v)$ vs. energy transfer (in $\left.\mathrm{cm}^{-1}\right)$.

Density functional theory (DFT) calculations: Discrete calculations on the structure of 4-(dimethylamino) benzaldehyde molecule were performed at the Perdew-Burke-Ernzerhof (PBE) [14] level of theory with the 6-311G(d,p) basis set using the Gaussian 09w program version [15]. Frequency calculations ensured that the optimized geometries were real minima (no negative eigenvalues) and provided the infrared and Raman intensities. The "OPT = ModRed" option of G09, by using a step size of $10^{\circ}$ to scan the relevant CNCH dihedral angle, allowed the potential energy functions for internal rotations to be obtained.

Periodic DFT calculations were performed using the plane-wave/pseudopotential method as implemented in the CASTEP code [16,17].The exchange-correlation energy in the calculation was described by the PBE functional [14]. The plane-wave cut-off energy of $830 \mathrm{eV}$ was taken with the $8 \times 4 \times 4$ Monkhorst-Pack grid for the electronic sampling of the Brillouin zone (BZ). An essential prerequisite for lattice dynamics calculations is the equilibrium structure, which was obtained by LBFGS geometry optimization, after which the residual forces were converged to zero within $0.005 \mathrm{eV} \cdot \mathrm{A}^{-1}$. From the reported crystal structure (CSD entry: ZZZMFY01) [18], the initial structure was taken and optimized, keeping constant the cell parameters. When using standard GGA functions, this is an important issue, as dispersion/van der Waals interactions description is defective, leading to unrealistic cell dimensions, as discussed elsewhere [19].

The diagonalization of dynamical matrices computed by the density-functional perturbation theory [20] with a coarse q-point grid of $4 \times 2 \times 1$, accounting for 12 q-points to sample the BZ, allowed for the calculation of phonon frequencies. Additionally, to the 
direct evaluation of frequencies and intensities at the $\Gamma$-point, the phonon dispersion was evaluated along a high-symmetry path throughout the BZ.

The atomic displacements for each normal mode (obtained from the CASTEP and G09 outputs) were used by the program Jmol [21] to visualize the modes, as well as to generate the INS spectrum through the program AbINS [22]. AbINS, an open-source package implemented as a plugin to the neutron data-analysis software Mantid [13] accounts for the neutron-scattering cross sections, overtones and combination modes. All that information, together with instrument-specific E-Q windows, produced a calculated INS spectrum that is easily compared with the experiment. For all the calculated spectra shown, it should be emphasized that the transition energies were not scaled.

\section{Results and Discussion}

\subsection{Molecular Geometry and Intermolecular Interactions}

Figure 1, left, presents the molecular structure of 4-(dimethylamino) benzaldehyde (4DMAB), along with the numbering scheme adopted in this work. The crystal structure of $4 \mathrm{DMAB}$ (monoclinic, space group P21/n, $\mathrm{Z}=8$ ) has been reported by Gao and Zhu [18] and by Vicente et al. [23]. Gao and Zhu [18] state that $4 \mathrm{DMAB}$ "crystallizes with two independent but essentially identical molecules in the asymmetric unit, which are linked via a $\mathrm{C}-\mathrm{H}^{\cdots} \pi$ interaction. In both molecules, the aldehyde and dimethylamine groups are essentially coplanar with the attached benzene ring. In the crystal structure, $\mathrm{C}-\mathrm{H} \cdots$ O hydrogen bonds link one type of independent molecules into a chain along the a axis. In addition, the structure is stabilized by $\pi$-stacking interactions involving the benzene rings.". The two molecules in the asymmetric unit are schematically represented in Figure 1, right, evidencing the $\mathrm{C}-\mathrm{H} \cdots \pi$ and $\mathrm{C}-\mathrm{H} \cdots \mathrm{O}$ contacts (along with the labels A and B for the two molecules and I-IV for the methyl groups).
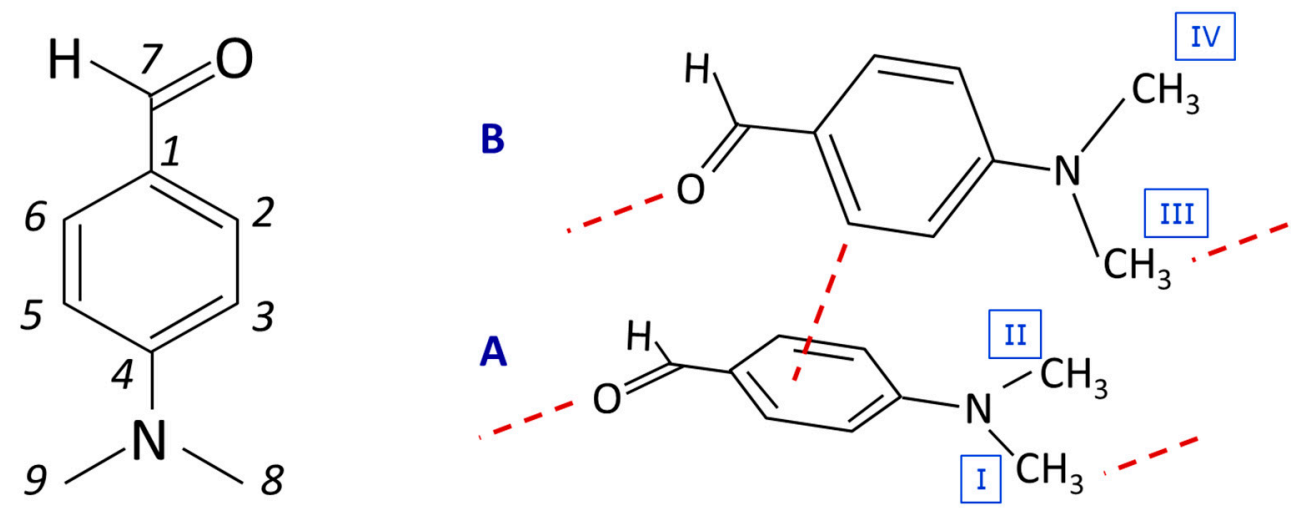

Figure 1. Representation of 4-(dimethylamino) benzaldehyde with the atom labelling used throughout the text (left) and a schematic representation of the crystal structure, evidencing the relevant intermolecular contacts present in the crystal (right). Molecules in the asymmetric unit are labelled A and B, and methyl groups are labelled I-IV.

A comparison between some selected geometric parameters obtained from X-ray [18], periodic calculations (CASTEP, see Experimental details) and discrete single-molecule calculations (G09, see Experimental details) can be found in Table 1. Overall, comparing the experimental and calculated crystal structures (X-ray vs. CASTEP), there is a good agreement. Deviations below 5\% and 2\% RMS are observed for the coordinates of all atoms (excluding hydrogen atoms) and for the bond lengths (excluding $\mathrm{C}-\mathrm{H}$ bonds), respectively. However, the presence of some well-known limitations of PBE calculations on CASTEP geometric parameters is verified. An overestimation of the $\mathrm{C}=\mathrm{O}$ bond length is clearly observed, leading to underestimation of the $\mathrm{C}=\mathrm{O}$ stretching mode, already discussed elsewhere $[19,24]$, and the pi-stacking distance evidences the foresee bias to large 
values. If not constrained by cell dimensions [19], this distance would deviate to physically meaningless values.

Table 1. Selected geometrical parameters of 4-(dimethylamino) benzaldehyde. Molecules in the asymmetric unit are labelled A and B, according to Figure 1.

\begin{tabular}{|c|c|c|c|c|c|}
\hline & X-ray (A) & X-ray (B) & CASTEP (A) & CASTEP (B) & G09 \\
\hline \multicolumn{6}{|l|}{ Bond length/pm } \\
\hline $\mathrm{C} 7=\mathrm{O}$ & 121.2 & 120.4 & 123.7 & 123.7 & 122.5 \\
\hline C1-C7 & 145.4 & 145.8 & 144.9 & 145.3 & 146.8 \\
\hline $\mathrm{C} 4-\mathrm{N}$ & 136.6 & 136.6 & 137.3 & 137.0 & 137.6 \\
\hline \multicolumn{6}{|l|}{ Bond angle $/^{\circ}$} \\
\hline $\mathrm{C} 1-\mathrm{C} 7-\mathrm{O}$ & 125.1 & 126.2 & 126.10 & 125.7 & 125.4 \\
\hline C4-N-C8 & 121.2 & 120.9 & 120.7 & 120.9 & 120.4 \\
\hline \multicolumn{6}{|l|}{ Dihedral angle ${ }^{\circ}$} \\
\hline $\mathrm{C} 2 \mathrm{C} 1-\mathrm{C} 7 \mathrm{O}$ & 1.4 & 1.6 & 1.5 & 0.3 & 0.0 \\
\hline C3C4-NC8 & -2.4 & 4.7 & -3.2 & 5.8 & 0.0 \\
\hline \multicolumn{6}{|l|}{ Distance/pm } \\
\hline $\mathrm{C} 8(\mathrm{H}) \cdots \mathrm{O}$ & 359.1 & 363.4 & 356.8 & 366.6 & - \\
\hline $\mathrm{C} 2(\mathrm{H}) \cdots \mathrm{Cg}^{1}$ & & 359.3 & & 363.1 & - \\
\hline$\pi \ldots \pi\left(\mathrm{C} 1 \cdots \mathrm{C} 4^{\prime}\right)^{2}$ & 370.4 & & 377.0 & & - \\
\hline
\end{tabular}

${ }^{1}$ Distance between $\mathrm{C} 2$ and the center of aromatic ring of the neighboring molecule; ${ }^{2}$ distance between $\mathrm{C} 1$ and $\mathrm{C} 4$ atoms of the $\pi$-stacking molecules (A).

Geometry obtained from single molecule (discrete) calculations (G09) at the PBE/6$311 \mathrm{G}(\mathrm{d}, \mathrm{p})$ level is in line with the one recently reported at the B3LYP/6-31G $(\mathrm{d}, \mathrm{p})$ level [25], and no large deviations from the CASTEP geometry are observed. For the isolated molecule, all non-hydrogen atoms are in the same molecular plane, with both substituents being coplanar with the aromatic ring. The dihedral angles shown in Table 1 are somewhat deviated from planarity, an effect of crystal packing already observed in similar systems $[4,9,10]$.

\subsection{Calculated vs. Experimental Spectra}

Figure 2 compares the experimental INS spectrum of $4 \mathrm{DMAB}$ (TOSCA) with the simulated INS spectra obtained from periodic (CASTEP) and discrete (G09) calculations, up to $1800 \mathrm{~cm}^{-1}$. Figure 3 presents a detailed view for the low-wavenumber region and its description without and with considering the effect of phonon dispersion (simulated spectra from $\Gamma$-point and with sampling over the Brillouin zone, respectively).

These figures evidence the excellent agreement between the experimental spectrum and the simulated spectrum derived from the periodic approach, in line with the results previously reported for similar benzaldehyde derivatives $[4,9,10]$. Even for the low-wavenumber modes (Figure 3), a region for which the computational description has been found to be more demanding $[10,26]$, the agreement between calculated and experimental profiles is very satisfactory. This agreement supports the reliable assignment of the vibrational modes of crystalline $4 \mathrm{DMAB}$, as discussed below.

Concerning the simulated spectrum from the discrete model (Figure 2, bottom), a reasonable agreement with the experimental spectrum is observed but only from ca. $700 \mathrm{~cm}^{-1}$ upwards, evidencing the difficulties of the single molecule approach to describe the crystalline structure. In the region below ca. $700 \mathrm{~cm}^{-1}$, the agreement is clearly unsatisfactory in both the position and intensity of the calculated bands. Of course, in the region where the contributions from collective modes are expected to prevail $\left(\mathrm{ca} .<100 \mathrm{~cm}^{-1}\right)$, the calculated spectrum for a single molecule is meaningless. 


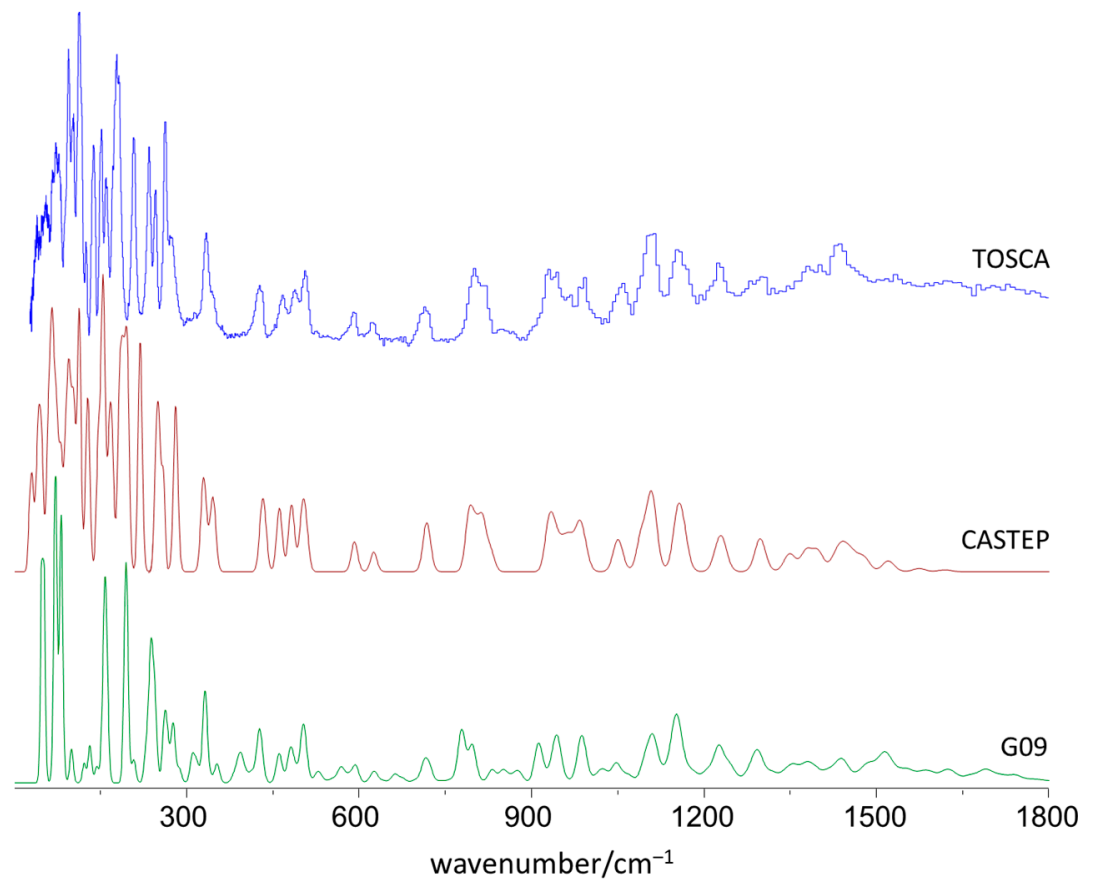

Figure 2. The INS spectra of 4-(dimethylamino) benzaldehyde in the $25-1800 \mathrm{~cm}^{-1}$ range: experimental (top), simulated from periodic calculations (middle) and from single-molecule discrete calculations (bottom).

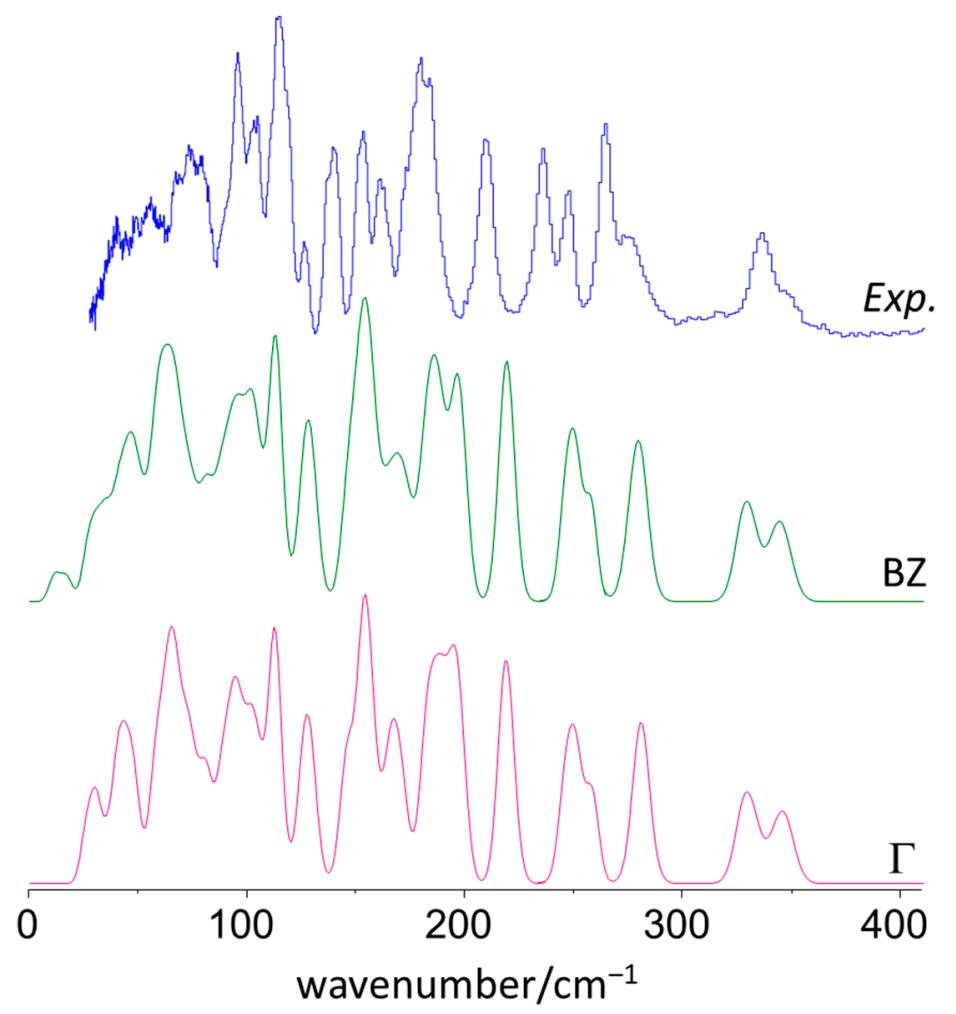

Figure 3. Experimental spectrum of 4-(dimethylamino) benzaldehyde in the range below $300 \mathrm{~cm}^{-1}$ (top) compared with the corresponding INS spectra calculated at the $\Gamma$-point $(\Gamma$, bottom) and averaged by the dispersion throughout the Brillouin zone (BZ, middle).

The crystallographic unit cell of 4DMAB (22 atoms per isolated molecule) contains 176 atoms. A total of 525 optical phonon modes are expected: 480 vibrational modes from 
the eight molecules (120 Ag + $120 \mathrm{Au}+120 \mathrm{Bg}+120 \mathrm{Bu})$ and 45 external modes describing translations and rotations (21 translational modes plus 24 librational modes, $12 \mathrm{Ag}+11 \mathrm{Au}$ $+11 \mathrm{Bg}+11 \mathrm{Bu})$.

From the 60 normal vibrational modes of each individual molecule, 30 are related to the phenyl ring, six to the aldehyde group and the remaining 24 to the dimethylamine fragment. The ring-related modes can be described as 12 stretching modes $(6 \times v C C$, $4 \times v \mathrm{CH}$ and $2 \times v \mathrm{C}-\mathrm{R} ; \mathrm{R}=$ substituents, $\left.\mathrm{CHO} / \mathrm{N}\left(\mathrm{CH}_{3}\right)_{2}\right)$, nine in-plane deformation modes $(3 \times \alpha$ ring, $4 \times \beta \mathrm{CH}$ and $2 \times \beta \mathrm{C}-\mathrm{R})$ and nine out-of-plane deformation modes $(3 \times \delta$ ring, $4 \times \gamma \mathrm{CH}$ and $2 \times \gamma \mathrm{C}-\mathrm{R})$. The six modes related to the aldehyde group can be described as two stretching modes $(v \mathrm{C}=\mathrm{O}, v \mathrm{C}-\mathrm{H})$, two in-plane deformation modes $(\beta C=\mathrm{O}, \beta \mathrm{C}-\mathrm{H})$, and two out-of-plane deformation modes $(\gamma \mathrm{C}=\mathrm{O}, \gamma \mathrm{C}-\mathrm{H})$, one of which is, in fact, the torsional mode $(\tau \mathrm{C}-\mathrm{CHO})$. The 24 modes related to the dimethylamine group can be described in terms of six NC2 modes (analogous to the 6 CHO modes) and of the well-known $2 \times 9-\mathrm{CH}_{3}$ modes (three stretching modes, five deformation modes and one torsional mode for each methyl group).

In INS, due to the absence of selection rules, all vibrational modes are permitted. However, since the signal intensity is highly dependent on the motions of hydrogen atoms (hydrogen atoms present the largest scattering cross-section and the largest displacements during vibration), only some modes have measurable intensity. This includes the lowfrequency/high-amplitude vibrational modes, an advantage of INS relative to its optical counterparts. Table 2 presents the observed and calculated band maxima and their approximate descriptions in terms of molecular vibrations. The "approximate description" of the normal modes refers to the most relevant contribution to each normal mode, despite their mixed nature resulting from coupling between oscillators. This description is assumed to be the most useful alternative to the more rigorous but often cumbersome description based on the potential energy distribution (PED), as discussed elsewhere [19].

Table 2. Experimental and calculated INS maxima for 4-(dimethylamino) benzaldehyde with vibrational mode assignments.

\begin{tabular}{ccc}
\hline $\begin{array}{c}\text { Calculated } \\
\text { (CASTEP) }^{\mathbf{1}}\end{array}$ & $\begin{array}{c}\text { Experimental } \\
\text { (TOSCA) }\end{array}$ & $\begin{array}{c}\text { Approximate } \\
\text { Description }\end{array}$ \\
\hline 1479 & 1473 & $\beta \mathrm{CH}_{3}$ \\
\hline 1442 & 1443 & $\beta \mathrm{CH}_{3}$ \\
\hline 1394 & 1408 & $\beta \mathrm{CH}_{3}$ \\
\hline 1382 & 1388 & $\beta \mathrm{CH}(=\mathrm{O})$ \\
\hline 1298 & 1309 & $\beta \mathrm{CH}$ \\
\hline 1229 & 1234 & $\gamma \mathrm{C}-\mathrm{CHO}$ \\
\hline 1156 & 1161 & $\beta \mathrm{CH}$ \\
\hline 1108 & 1116 & $\beta \mathrm{CH}$ \\
\hline 1089 & 1116 & rock CH 3 \\
\hline 1050 & 1065 & rock $\mathrm{CH} H_{3}$ \\
\hline 985 & 993 & $\gamma \mathrm{CH}(=\mathrm{O})$ \\
\hline 963 & 970 & $\gamma \mathrm{CH}$ \\
\hline 940 & 951 & $\gamma \mathrm{CH}$ \\
\hline 930 & 933 & $v \mathrm{~N}-(\mathrm{CH})_{2}$ \\
\hline 814 & 825 & $\gamma \mathrm{CH}$ \\
\hline 795 & 806 & $\gamma \mathrm{CH}$ \\
\hline
\end{tabular}


Table 2. Cont.

\begin{tabular}{|c|c|c|}
\hline $\begin{array}{c}\text { Calculated } \\
\text { (CASTEP) }^{1}\end{array}$ & $\begin{array}{c}\text { Experimental } \\
\text { (TOSCA) }\end{array}$ & $\begin{array}{l}\text { Approximate } \\
\text { Description }\end{array}$ \\
\hline 718 & 719 & $\delta$ ring \\
\hline 625 & 630 & $\alpha$ ring \\
\hline 593 & 594 & $\alpha$ ring \\
\hline 505 & 509 & $\delta$ ring \\
\hline 483 & 490 & $\beta \mathrm{N}-\left(\mathrm{CH}_{3}\right)_{2}$ \\
\hline 461 & 470 & $\beta \mathrm{N}-\left(\mathrm{CH}_{3}\right)_{2}$ \\
\hline 433 & 431 & $\delta$ ring \\
\hline 345 & 348 & $\alpha$ ring \\
\hline 329 & 336 & $\gamma$ ring- $\mathrm{CHO}$ \\
\hline- & 275 & (see text) \\
\hline 281 & 265 & $\beta$ ring- $\mathrm{N}\left(\mathrm{CH}_{3}\right)_{2}$ \\
\hline 255 & 247 & $\gamma$ ring- $\mathrm{N}\left(\mathrm{CH}_{3}\right)_{2}$ \\
\hline 251 & 237 & \\
\hline 219 & 210 & $\tau \mathrm{N}-\mathrm{CH} 3$ \\
\hline 195 & 184 & \\
\hline 189 & 180 & $\tau \mathrm{N}-\mathrm{CH}_{3}$ \\
\hline 182 & 172 & \\
\hline 168 & 162 & $\beta$ ring- $\mathrm{CHO}$ \\
\hline 155 & 153 & $\gamma \mathrm{N}-\left(\mathrm{CH}_{3}\right)_{2}$ \\
\hline 146 & 138 & \\
\hline 128 & 128 & $\tau \mathrm{C}-\mathrm{N}\left(\mathrm{CH}_{3}\right)_{2}$ \\
\hline 113 & 115 & \\
\hline 101 & 105 & $\tau \mathrm{C}-\mathrm{CHO}$ \\
\hline 95 & 90 & Libration \\
\hline 83 & 75 & Libration \\
\hline 66 & 67 & Libration \\
\hline 49 & 51 & Translation \\
\hline 43 & 39 & Translation \\
\hline
\end{tabular}

${ }^{1}$ Maxima in the INS simulated spectrum. $\nu, \alpha, \beta, \gamma$ and $\tau$ stand for stretching, in-plane deformation, out-of-plane deformation and torsion modes, respectively.

Apart from the external or collective modes, phonon modes in $4 \mathrm{DMAB}$ crystal were found to be grouped in combinations of eight identical molecular normal modes-generally, combinations of four A-type molecules and four B-type molecules-giving rise to the observed INS band maxima. In that sense, bands in Table 2 are described in terms of molecular normal modes of vibration. However, since $4 \mathrm{DMAB}$ crystal results from the packing of molecules contained in nearly orthogonal planes (namely, molecules linked via a C-H $\cdots \pi$ interaction), mixing between in-plane and out-of-plane molecular modes is observed. In a few cases, the phonon mode includes an in-plane mode of one B-type molecule and an out-of-plane mode of the neighboring A-type molecule. Figure 4 illustrates the situation for one of the phonon modes contributing to the $719 \mathrm{~cm}^{-1}$ band, which is nominally described as "ring out-of-plane deformation" but includes contributions from the "ring in-plane deformation" of the B-type molecules. Since the molecules are in orthogonal planes, all the atoms in both molecules move along the same "direction" (more rigorously, move in parallel planes). 


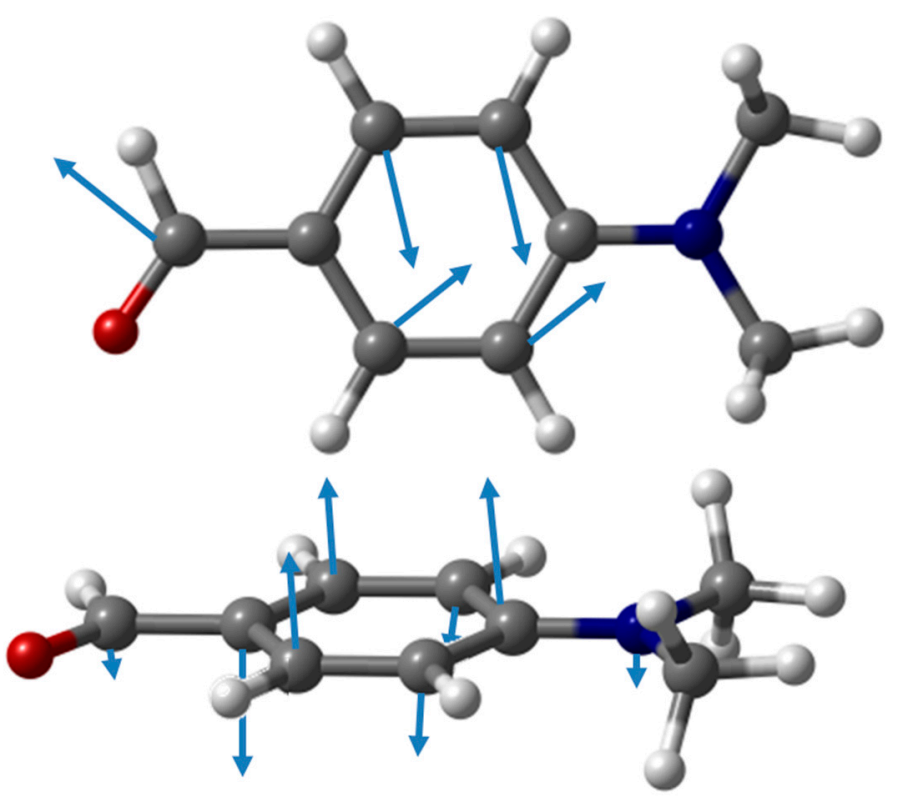

Figure 4. Atomic displacements of heavy atoms in one of the phonon modes contributing to the band at ca. $719 \mathrm{~cm}^{-1}$. The large displacements of hydrogen atoms were omitted for clarity.

A straightforward validation of the model is provided by the excellent description of the INS spectrum obtained from the periodic calculations, thus allowing a trustful assignment of vibrational modes of crystalline $4 \mathrm{DMAB}$. The vibrational assignment of IR and Raman spectra of $4 \mathrm{DMAB}$ has been reported, based on an empirical force-field normal coordinate analysis [27] and on discrete calculations at the B3LYP/6-31G(d,p) level [15]. A partial assignment was also reported by the authors in an attempt to describe the spectrum of the crystalline samples from the sum of dimer contributions [28].

There is a general concordance among assignments in the medium-wavenumber region, with a few discrepancies concerning the substituent groups. For the most relevant discrepancies, which occur for the low-wavenumber region, the combination of periodic calculations and INS spectrum provides clarification, as discussed below.

For instance, Rocha et al. [25] relate the $177 \mathrm{~cm}^{-1}$ band in the Far-IR spectrum to the torsion of the -CHO group, a mode assigned to the Far-IR band at ca. $109 \mathrm{~cm}^{-1}$ by Kushto and Jagodzinski [27]. Rocha et al. also report and assign a Far-IR band at $247 \mathrm{~cm}^{-1}$ to the in-plane bending motion of the dimethylamino substituent, a band not identified by Kushto and Jagodzinski in their work. According to CASTEP calculations (Table 2), the torsional - $\mathrm{CHO}$ mode is better assigned to the INS band at $105 \mathrm{~cm}^{-1}$, in agreement with Kushto and Jagodzinski, and the band at $247 \mathrm{~cm}^{-1}$ is better related with the out-of-plane bending motion of the dimethylamino substituent. However, since both works ignore the intermolecular interactions in the crystal and Kushto and Jagodzinski did not include the internal rotations of the methyl groups and the dimethylamino group in their calculations, their analysis of the low-wavenumber region is necessarily incomplete and defective.

One of the most evident effects of the intermolecular interactions due to crystal packing is the factor group splitting. In fact, the factor group splitting for a few modes in $4 \mathrm{DAMB}$ crystal observed in the INS spectra is foreseen by periodic calculations. Factor group splitting, as would be expected, is present in a crystal cell with eight molecules (eight molecular modes are expected to combine into eight phonon modes), as its magnitude is influenced by the magnitude of the intermolecular forces in the crystal. For most of the vibrational modes, the factor-group components are not separated (considering the spectral resolution) and do not influence the observed spectra.

As can be seen in Table 2, crystal splitting is predicted to have observable magnitude for the phonon modes below $350 \mathrm{~cm}^{-1}$ of $4 \mathrm{DMAB}$ but only in the modes related to the dimethylamino group. In fact, none of the aldehyde-related modes (C-CHO out-of-plane 
bend, in-plane bend, and torsion) presents measurable splitting. This is a particular feature of $4 \mathrm{DMAB}$ since for the other previously reported 4-substituted benzaldehyde crystals $[4,9,10]$, factor group splitting was observed for the - $\mathrm{CHO}$ modes (at least for the -CHO torsional mode).

On the other hand, the splitting is clearly predicted and observed for the corresponding $-\mathrm{N}\left(\mathrm{CH}_{3}\right)_{2}$ modes, plus the $\mathrm{CH}_{3}$ torsional modes. The notable exception is the in-plane bending of the $\mathrm{C}-\mathrm{N}\left(\mathrm{CH}_{3}\right)_{2}$, for which a possible splitting is observed and assigned, as observed in Table 2, although it is not predicted from theoretical calculations. For this pair of bands, 265 and $275 \mathrm{~cm}^{-1}$, the alternative assignment of a single band plus a 0-2 transition cannot be excluded.

Figure 5 illustrates the phonon distribution in the calculated INS below $300 \mathrm{~cm}^{-1}$, with special emphasis on the bands ascribed to methyl torsional motions. Each torsional mode is labelled according to its position, as detailed in Figure 1. The labels I-IV distinguish the methyl groups according to their positions in the asymmetric unit. The methyl groups are all inequivalent due to their neighboring contacts (as can be seen in Figure 1). For instance, methyl group I is engaged in $\mathrm{C}-\mathrm{H} \cdots \mathrm{O}$ bonding, while methyl group II is not. Identically, methyl group III presents a shorter $\mathrm{C}-\mathrm{H} \cdots \mathrm{O}$ contact than methyl group IV, which, in turn, is closer to the $\pi$-system.

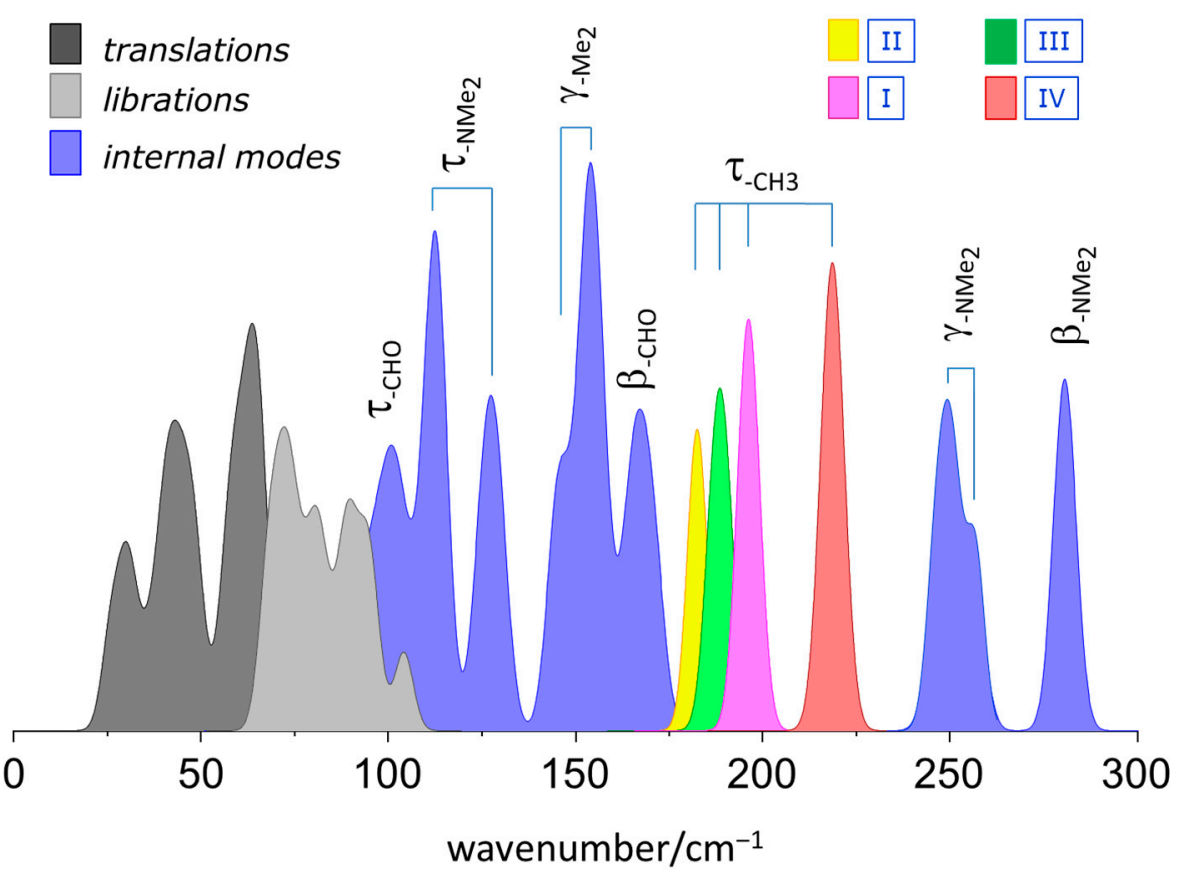

Figure 5. Pictorial description of the calculated band maxima in the low-wavenumber region (from periodic-DFT calculations). External modes (translations and librations) in grey, internal modes in blue, except for the $\mathrm{CH}_{3}$ torsional modes, with a differentiated color code. The $\mathrm{CH}_{3}$ torsional modes are labelled I-IV, according to Figure 1.

The methyl torsion with the lowest wavenumber is II, while that with the highest energy is III. Torsional modes I and II are highly localized in the corresponding methyl group, but modes III and IV are less localized and can alternatively be described in terms of (III + IV) symmetrical and asymmetrical combinations, respectively. The predicted frequencies and intensities of the methyl torsion modes are in very good agreement with the experimental frequencies and intensities, which suggests that a classical approach is enough to describe the behavior of methyl torsion in this system.

The torsional frequencies of the four methyl groups fall in a region of ca. $190 \pm 20 \mathrm{~cm}^{-1}$. According to the literature, torsional modes of methyl groups bonded to $\mathrm{sp}^{2}$ carbon atoms appear at $\leq 200 \mathrm{~cm}^{-1}$, while those bonded to $\mathrm{sp}^{3}$ carbon atoms occur at $\sim 250 \mathrm{~cm}^{-1}$ [29]. 
For methoxybenzaldehydes [10], it was found that methyl groups bonded to $\left(\mathrm{sp}^{3}\right)$ oxygen atoms also occur at $250 \mathrm{~cm}^{-1}$, thus pointing to a similar nature of the potential energy barrier for the rotation along the $\mathrm{C}\left(\mathrm{sp}^{3}\right)-\mathrm{CH}_{3}$ and $\mathrm{O}-\mathrm{CH}_{3}$ bonds. The present work places the $\mathrm{N}-\mathrm{CH}_{3}$ torsional motion close to the $\mathrm{C}\left(\mathrm{sp}^{2}\right)-\mathrm{CH}_{3}$ case, which may suggest a different tendency between oxygen and nitrogen atoms. It should be mentioned, however, that the planarity of $4 \mathrm{DMAB}$ can be ascribed to a resonance structure with an $\mathrm{sp}^{2}$ nitrogen atom. To the best of our knowledge, the data for methyl torsional motions in non-aromatic amines are scarce, but in the case of the quaternary ammonium ion choline, with methyl groups bound to an $\mathrm{sp}^{3}$ nitrogen atom, torsional modes in the range of $250-330 \mathrm{~cm}^{-1}$ have been observed in the INS spectrum [30]. On the whole, these observations point to the importance of hybridization of the atom to which methyl group is bound as the determining factor for the torsional barrier of the methyl groups.

For the isolated molecules, there is no significant difference between the two methyl groups, and the potential energy scan for the rotation of a single methyl group yields a $\mathrm{V}_{3}$ potential of ca. $430 \mathrm{~cm}^{-1}$ (at PBE/6-311G** level). This is considerably lower than the values reported for $\mathrm{N}$-methylaniline, obtained from similar discrete DFT calculations (ca. $\left.760 \mathrm{~cm}^{-1}\right)$ [31] or derived from microwave spectroscopy $\left(975 \mathrm{~cm}^{-1}\right)$ [32]. The planarity of the $\mathrm{N}\left(\mathrm{CH}_{3}\right)_{2}$ moiety and the presence of a second methyl group are expected to contribute to this difference in barrier-energy heights.

\section{Conclusions}

INS spectroscopy offers unique access to the low-wavenumber modes, particularly those involving large atomic displacements of hydrogen atoms, and has a synergic combination with periodic DFT calculations. All the gathered information in this work provides a good basis for interpreting the vibrational spectra and assessing the structure and dynamics of $4 \mathrm{DMAB}$ in the crystal form, given the excellent agreement between calculated and experimental INS spectra.

The external phonon modes of crystalline $4 \mathrm{DMAB}$ are quite well described by the simulated spectrum, a somewhat unusual situation, due to de limitations of periodic calculations (in short, low-wavenumber modes are known to collect calculations errors). In what concerns the dynamics of the low-wavenumber/high-amplitude molecular motions, the behavior of the $-\mathrm{CHO}$ and $-\mathrm{N}\left(\mathrm{CH}_{3}\right)_{2}$ substituents is dissimilar. Crystal field splitting is only observed for the modes assigned to the dimethylamino group and not for the aldehyde-related modes. It should be emphasized that the use of a simplified approximate description of the normal modes is not intended to hide the complex nature of some vibrational motions. Actually, in most of the low-wavenumber vibrations involving the substituent groups, the single-oscillator mode is more an exception than a rule. Hence, the description "-CHO modes" or "-N( $\left(\mathrm{CH}_{3}\right)_{2}$ modes" only identifies a dominant contribution to a complex vibrational mode.

The torsional frequencies of the four methyl groups fall in a region of ca. $190 \pm 20 \mathrm{~cm}^{-1}$, close to the range of values observed for methyl groups bond to unsaturated carbon atoms. Comparing the INS data gathered for $\mathrm{C}-\mathrm{CH}_{3}, \mathrm{O}_{-} \mathrm{CH}_{3}$ and N-CH , the previously formulated general rule concerning torsional frequency of methyl groups [29] may be upgraded to: "torsional modes of methyl groups bonded to sp' atoms occur at $\leq 200 \mathrm{~cm}^{-1}$, while those bonded to $\mathrm{sp}^{3}$ atoms appear at $\sim 250 \mathrm{~cm}^{-1 "}$.

Author Contributions: Formal analysis, P.J.A.R.-C.; investigation, M.M.N. (INS, CASTEP), P.J.A.R.-C. (G09), P.D.V. (INS); writing—original draft preparation, M.M.N.; writing—review and editing, P.D.V. and P.J.A.R.-C.; supervision, P.J.A.R.-C.; funding acquisition, M.M.N. All authors have read and agreed to the published version of the manuscript.

Funding: This work received financial support from the Portuguese Foundation for Science and Technology (FCT) - project CICECO, Aveiro Institute of Materials, POCI-01-0145-FEDER-007679 (UID/CTM/50011/2013) - financed by national funds through the FCT/MEC and when appropriate co-financed by FEDER under the PT2020 Partnership Agreement. Experiments at the ISIS 
Neutron and Muon Source were supported by beamtime allocation RB15119 from the Science and Technology Facilities Council. FCT is gratefully acknowledged for a researcher contract to M.M.N. (IF/01468/2015) under the program IF 2015.

Data Availability Statement: Data from the neutron scattering experiments can be accessed freely at https: / /data.isis.stfc.ac.uk/\#/browse/facility/ISIS/instrument/17/facilityCycle/23/investigation/ 14824759 / dataset (accessed on 6 November 2021).

Acknowledgments: The STFC Rutherford Appleton Laboratory is thanked for access to neutron beam facilities. CASTEP calculations were made possible due to the computing resources provided by STFC Scientific Computing Department's SCARF cluster.

Conflicts of Interest: The authors declare no conflict of interest.

\section{References}

1. Araujo, C.; Freire, C.S.R.; Nolasco, M.M.; Ribeiro-Claro, P.J.A.; Rudić, S.; Silvestre, A.J.D.; Vaz, P.D. Hydrogen bond dynamics of cellulose through Inelastic Neutron Scattering Spectroscopy. Biomacromolecules 2018, 19, 1305-1313. [CrossRef] [PubMed]

2. Araujo, C.F.; Nolasco, M.M.; Ribeiro-Claro, P.J.A.; Rudić, S.; Silvestre, A.J.D.; Vaz, P.D.; Sousa, A.F. Inside PEF: Chain conformation and dynamics in crystalline and amorphous domains. Macromolecules 2018, 51, 3515-3526. [CrossRef]

3. Parker, S.F.; Butler, I.R. Synthesis, computational studies, inelastic neutron scattering, infrared and raman spectroscopy of ruthenocene. Eur. J. Inorg. Chem. 2019, 2019, 1142-1146. [CrossRef]

4. Nolasco, M.M.; Araujo, C.F.; Vaz, P.D.; Amado, A.M.; Ribeiro-Claro, P. Vibrational dynamics of crystalline 4-phenylbenzaldehyde from INS spectra and periodic DFT calculations. Molecules 2020, 25, 1374. [CrossRef] [PubMed]

5. Bilski, P.; Druzbicki, K.; Jenczyk, J.; Mielcarek, J.; Wasicki, J. Molecular and vibrational dynamics in the cholesterol-lowering agent lovastatin: Solid-state NMR, inelastic neutron scattering, and periodic DFT study. J. Phys. Chem. B 2017, 121, $2776-2787$. [CrossRef]

6. Pawlukojć, A.; Hetmańczyk, Ł. INS, DFT and temperature dependent IR studies on dynamical properties of acetylcholine chloride. Vib. Spectrosc. 2016, 82, 37-43. [CrossRef]

7. Drużbicki, K.; Mikuli, E.; Pałka, N.; Zalewski, S.; Ossowska-Chruściel, M.D. Polymorphism of resorcinol explored by complementary vibrational spectroscopy (FT-RS, THz-TDS, INS) and first-principles solid-state computations (Plane-Wave DFT). J. Phys. Chem. B 2015, 119, 1681-1695. [CrossRef] [PubMed]

8. Zhong, L.; Parker, S.F. Structure and vibrational spectroscopy of methanesulfonic acid. R. Soc. Open Sci. 2018, 5, 181363. [CrossRef] [PubMed]

9. Ribeiro-Claro, P.J.A.; Vaz, P.D.; Nolasco, M.M.; Araujo, C.F.; Gil, F.P.S.C.; Amado, A.M. Vibrational dynamics of 4fluorobenzaldehyde from periodic DFT calculations. Chem. Phys. Lett. X 2019, 737, 100006. [CrossRef]

10. Ribeiro-Claro, P.J.A.; Vaz, P.D.; Nolasco, M.M.; Gil, F.P.S.C.; de Carvalho, L.A.E.; Marques, M.P.M.; Amado, A.M. New Insights on the vibrational dynamics of 2-Methoxy-, 4-Methoxy- and 4-Ethoxy-Benzaldehyde from INS Spectra and Periodic DFT calculations. Materials 2021, 14, 4561. [CrossRef]

11. Parker, S.F.; Lennon, D.; Albers, P.W. Vibrational spectroscopy with neutrons: A review of new directions. Appl. Spectrosc. 2011, 65, 1325-1341. [CrossRef]

12. Parker, S.F.; Fernandez-Alonso, F.; Ramirez-Cuesta, A.J.; Tomkinson, J.; Rudic, S.; Pinna, R.S.; Gorini, G.; Fernandez Castanon, J. Recent and future developments on TOSCA at ISIS. J. Phys. Conf. Ser. 2014, 554, 012003. [CrossRef]

13. Arnold, O.; Bilheux, J.C.; Borreguero, J.M.; Buts, A.; Campbell, S.I.; Chapon, L.; Doucet, M.; Draper, N.; Leal, R.F.; Gigg, M.A.; et al. Mantid-Data analysis and visualization package for neutron scattering and mu SR experiments. Nucl. Instrum. Methods Phys. Res. A 2014, 764, 156-166. [CrossRef]

14. Perdew, J.P.; Burke, K.; Ernzerhof, M. Generalized gradient approximation made simple. Phys. Rev. Lett. 1996, 77, 3865-3868. [CrossRef] [PubMed]

15. Frisch, M.J.; Trucks, G.W.; Schlegel, H.B.; Scuseria, G.E.; Robb, M.A.; Cheeseman, J.R.; Scalmani, G.; Barone, V.; Mennucci, B.; Petersson, G.A.; et al. Gaussian 09, Revision A. Available online: https:/ / www.scienceopen.com/document?vid=6be7271f-f651-4 64b-aee6-ef20b0743b6b (accessed on 6 November 2021).

16. Clark, S.J.; Segall, M.D.; Pickard, C.J.; Hasnip, P.J.; Probert, M.J.; Refson, K.; Payne, M.C. First principles methods using CASTEP. Z. Fur Krist. 2005, 220, 567-570. [CrossRef]

17. Refson, K.; Tulip, P.R.; Clark, S.J. Variational density-functional perturbation theory for dielectrics and lattice dynamics. Phys. Rev. $B$ 2006, 73, 155114. [CrossRef]

18. Gao, B.; Zhu, J.-L. 4-(Dimethylamino) benzaldehyde. Acta Crystallogr. Sect. E Struct. Rep. Online 2008, 64, o1182. [CrossRef]

19. Ribeiro-Claro, P.J.A.; Vaz, P.D.; Nolasco, M.M.; Amado, A.M. Understanding the vibrational spectra of crystalline isoniazid: Raman, IR and INS spectroscopy and solid-state DFT study. Spectrochim. Acta Part A Mol. Biomol. Spectrosc. 2018, 204, 452-459. [CrossRef]

20. Baroni, S.; de Gironcoli, S.; Dal Corso, A.; Giannozzi, P. Phonons and related crystal properties from density-functional perturbation theory. Rev. Mod. Phys. 2001, 73, 515-562. [CrossRef] 
21. Jmol. An Open-Source Java Viewer for Chemical Structures in 3D. Available online: http://jmol.sourceforge.net/ (accessed on 6 November 2021).

22. Dymkowski, K.; Parker, S.F.; Fernandez-Alonso, F.; Mukhopadhyay, S. AbINS: The modern software for INS interpretation. Phys. $B$ Condens. Matter 2018, 551, 443-448. [CrossRef]

23. Edith, A.K.; Vicente, H.; Graca, M.; Fronczek, F.R. 4-(dimethylamino) benzaldehyde. CCDC 2023780. Exp. Cryst. Struct. Determ. 2020. [CrossRef]

24. Drużbicki, K.; Mielcarek, J.; Kiwilsza, A.; Toupet, L.; Collet, E.; Pajzderska, A.; Wąsicki, J. Computationally assisted (solid-state density functional theory) structural (X-ray) and vibrational spectroscopy (FT-IR, FT-RS, TDs-THz) characterization of the cardiovascular drug lacidipine. Cryst. Growth Des. 2015, 15, 2817-2830. [CrossRef]

25. Rocha, M.; Di Santo, A.; Marcelo Arias, J.; Gil, D.M.; Ben Altabef, A. Ab-initio and DFT calculations on molecular structure, NBO, HOMO-LUMO study and a new vibrational analysis of 4-(Dimethylamino) Benzaldehyde. Spectrochim. Acta Part A Mol. Biomol. Spectrosc. 2015, 136, 635-643. [CrossRef]

26. Adilina, I.B.; Aulia, F.; Fitriady, M.A.; Oemry, F.; Widjaya, R.R.; Parker, S.F. Computational and spectroscopic studies of carbon disulfide. Molecules 2020, 25, 1901. [CrossRef]

27. Kushto, G.P.; Jagodzinski, P.W. Vibrational spectra and normal coordinate analysis of 4-(dimethylamino) benzaldehyde and selected isotopic derivatives. Spectrochim. Acta Part A Mol. Biomol. Spectrosc. 1998, 54, 799-819. [CrossRef]

28. Ribeiro-Claro, P.J.A.; Vaz, P.D.; Nolasco, M. Crystal structure landscapes from combined vibrational spectroscopy and ab initio calculations: 4-(Dimethylamino)benzaldehyde. J. Mol. Struct. Theochem. 2010, 946, 65-69. [CrossRef]

29. Zachariou, A.; Hawkins, A.P.; Collier, P.; Howe, R.F.; Lennon, D.; Parker, S.F. The methyl torsion in unsaturated compounds. ACS Omega 2020, 5, 2755-2765. [CrossRef] [PubMed]

30. Araujo, C.F.; Coutinho, J.A.P.; Nolasco, M.M.; Parker, S.F.; Ribeiro-Claro, P.J.A.; Rudić, S.; Soares, B.I.G.; Vaz, P.D. Inelastic neutron scattering study of reline: Shedding light on the hydrogen bonding network of deep eutectic solvents. Phys. Chem. Chem. Phys. 2017, 19, 17998-18009. [CrossRef]

31. Emel'yanenko, V.N.; Pimerzin, A.A.; Turovtsev, V.V.; Verevkin, S.P. Benchmark thermochemistry of N-Methylaniline. J. Phys. Chem. A 2015, 119, 2142-2152. [CrossRef]

32. Fujitake, M.; Aoyama, J.; Ohashi, N. Fourier-transform microwave spectroscopy of N-methylaniline. J. Mol. Spectrosc. 2006, 235, 27-34. [CrossRef] 